\title{
C stars as kinematic probes of the Milky Way disk from 9 to $15 \mathrm{kpc}^{\star}$
}

\author{
S. Demers ${ }^{1, \star \star}$ and P. Battinelli ${ }^{2}$ \\ 1 Département de Physique, Université de Montréal, CP 6128, Succursale Centre-Ville, Montréal, Qc, H3C 3J7, Canada \\ e-mail: demers@astro.umontreal.ca \\ 2 INAF, Osservatorio Astronomico di Roma, Viale del Parco Mellini 84, 00136 Roma, Italy \\ e-mail: battinel@oarhp1.rm.astro.it
}

Received 23 April 2007 / Accepted 27 June 2007

\begin{abstract}
Context. The availability, from 2MASS, of a large homogeneous sample of Galactic C stars and the recognition that their absolute magnitude can be accurately determined offer the possibility to use them as kinematical probes to investigate motions in the thin or thick disks.

Aims. Determine the radial velocities for $70 \mathrm{C}$ stars, a few degrees from the Galactic plane and distributed in longitudes from $60^{\circ}$ to $220^{\circ}$.

Methods. Spectra, with a resolution of 4300, were obtained with the DAO $1.8 \mathrm{~m}$ telescope during 6 beautiful nights in October 2006. Results. The rotation velocities of $\mathrm{C}$ stars with $60^{\circ}<\ell<150^{\circ}$ suggest a flat rotation curve to $15 \mathrm{kpc}$. A number of stars have velocities that do not fit the thin disk rotation. Some of them, toward $\ell=200^{\circ}$ are most probably members of the Canis Major overdensity.

Conclusions. Effort should be made to extend the rotation curve to more that $20 \mathrm{kpc}$.
\end{abstract}

Key words. stars: carbon - stars: kinematics - Galaxy: disk - Galaxy: kinematics and dynamics

\section{Introduction}

The importance of galactic potentials in shaping the chemodynamical evolution of stellar populations is well known. Unfortunately, the surface mass distribution of the Milky Way is still poorly constrained, even while our knowledge of Galactic stellar abundance distributions grows ever more detailed. One reason for this shortcoming is the difficulty of determining the Galactic rotation curve in the outer disk.

Recently, Carignan et al. (2006) have published the HI rotation curve of M 31 up to a radius of $35 \mathrm{kpc}$. Battinelli \& Demers (2005a) discovered numerous $\mathrm{C}$ stars in the outer disk of M 31 reaching $40 \mathrm{kpc}$. On the other hand, the Galactic rotation curve well beyond the solar circle, is in a rather poor state. Radio astronomers indeed cannot do HI tangent point analysis outside the solar circle and other unsatisfactory methods have been considered. Merrifield (1992) published a rotation curve to a galactocentric distance of $20 \mathrm{kpc}$ using the thickness of the HI disk as a distance indicator. His curve agrees with the planetary nebulae data of Sneider \& Terzian (1983).

Recently, Frinchaboy \& Majewski (2005) have started a long-term project of open cluster distances and velocities determinations with the aim of extending the rotation curve of the Galaxy in the direction of the anticenter. An alternative to the above methods is the use of $\mathrm{C} \mathrm{N}$-type stars as kinematical probes. They are intrinsically bright, $\left\langle M_{I}\right\rangle=-4.6$ (Battinelli \& Demers 2005b). Moreover, these intermediate-age stars are old enough to have lost memories of any systematic velocity with

${ }^{\star}$ Full Tables 1 and 4 are only available in electronic form at http: //www . aanda.org

$\star \star$ Guest investigators, Dominion Astrophysical Observatory, Herzberg Institute of Astrophysics, National Research Council of Canada. which they might have been born but still relatively young, with smaller random velocities than older tracers (e.g. planetary nebulae). They are believed to be members of the thin disk population (e.g. Feast et al. 2006).

In the late 80's, Aaronson and collaborators recognized that C stars could be useful bright kinematical probes (Aaronson et al. 1989, 1990). They collected hundreds of radial velocities of disk $\left(b \approx 0^{\circ}\right) \mathrm{C}$ stars, with $J H K$ photometry. The fact that these $\mathrm{C}$ stars were extremely unevenly distributed in galactic latitude made the interpretation of their results unreliable (Schechter et al. 1988). A study of the kinematics of the C stars toward the anticenter was published by Metzger \& Schechter (1994). Beside the untimely death of Aaronson we believe there are two main reasons why these data never yielded significant insights in the kinematics of the outer disk: 1) the selection of $\mathrm{C}$ stars from objective-prism spectra leads to highly inhomogeneous samples containing faint bluish $\mathrm{C}$ stars mixed with brighter genuine N-type C stars (see Demers et al. 2002) thus artificially increasing the observed $\mathrm{K}$ absolute magnitude spread; 2 ) the estimate of distances and colors for stars with $b \approx 0^{\circ}$ requires a quite accurate knowledge of the extinction across the disk.

More recently, Nakashima et al. (2000) obtained the rotation curve of the outer disk using the radial velocity of a few C- and O-rich $\mathrm{SiO}$ maser emission Miras located very close to the Galactic plane. Absolute $I$ magnitudes were deduced from the Mira's $P-L$ relation. Not knowing individual reddening, the authors had to make assumptions regarding the reddening along the lines of sight. One other serious complication is the presence of circumstellar envelops around $\mathrm{SiO}$ maser Miras, this could very well affect their apparent magnitude thus making the $P-L$ relation somewhat problematic.

Today we are in a better position to re-attack this problem. 2MASS has provided us with reliable $J H K$ photometry of 
Table 1. C star candidates ${ }^{a}$.

\begin{tabular}{|c|c|c|c|c|c|c|c|c|c|c|}
\hline$\overline{\mathrm{id}}$ & \multicolumn{2}{|c|}{$\overline{\text { RA J2000 Dec }}$} & $\overline{\overline{K_{\mathrm{s}}}}$ & $\overline{\left(J-K_{\mathrm{s}}\right)}$ & $\overline{\left(H-K_{\mathrm{s}}\right)}$ & $\overline{\overline{E_{(B-V)}}}$ & $\bar{I}$ & $\overline{d_{\odot}}$ & $\overline{d_{\mathrm{GC}}}$ & $z$ \\
\hline 1 & 03537.5 & 580024.3 & 4.93 & 1.95 & 0.68 & 0.43 & 9.02 & 3.6 & 10.0 & -0.30 \\
\hline 2 & 04617.9 & 593740.1 & 4.91 & 1.89 & 0.62 & 0.58 & 9.10 & 3.3 & 9.8 & -0.19 \\
\hline 3 & 11852.8 & 580931.0 & 4.68 & 2.10 & 0.73 & 0.49 & 8.99 & 3.4 & 10.0 & -0.26 \\
\hline 4 & 12518.9 & 573918.0 & 5.19 & 2.01 & 0.65 & 0.56 & 9.48 & 4.0 & 10.5 & -0.34 \\
\hline 5 & 13416.1 & 574327.6 & 5.00 & 1.80 & 0.60 & 0.59 & 9.11 & 3.3 & 10.0 & -0.27 \\
\hline 6 & 14208.7 & 575532.1 & 6.25 & 1.73 & 0.57 & 0.51 & 10.20 & 5.8 & 12.2 & -0.43 \\
\hline 7 & 14358.3 & 58410 & 4.89 & 1.92 & 0.69 & 0.56 & 9.09 & 3.3 & 10.1 & -0.20 \\
\hline 8 & 15055.6 & 572851.0 & 5.54 & 1.62 & 0.54 & 0.32 & 9.19 & 4.3 & 10.9 & -0.34 \\
\hline 9 & 15215.5 & 565802.5 & 5.54 & 1.66 & 0.59 & 0.30 & 9.21 & 4.4 & 11.1 & -0.38 \\
\hline 10 & 20639.6 & 565106.2 & 4.89 & 2.13 & 0.85 & 0.43 & 9.16 & 3.9 & 10.6 & -0.30 \\
\hline
\end{tabular}

a Table 1 is presented in its entirety in the electronic edition of Astronomy \& Astrophysics. A portion is shown here for guidance regarding its form and content. Units of right ascensions are hours, minutes and seconds, and units of declination are degrees, arcminutes and arcseconds. Distances are in kpc.

spectroscopically known C stars, along with hundreds of fainter Galactic C stars (candidates); Schlegel et al. (1998) reddening maps are now in general use and are considered quite reliable as long as the extremely low galactic latitudes are avoided. Weinberg \& Nikolaev (2001) have shown that N-type C stars can be used, under certain colour restrictions, as reliable standard candles showing a small dispersion in $M_{K}$. More recently Mauron et al. (2004) followed a similar method to determine the distances for several halo $\mathrm{C}$ stars. This approach is preferable to the $P-L$ relation which requires the $\mathrm{C}$ star to be a Mira of known period.

\section{C star candidate selection}

In recent years, several authors have used near-IR photometry to select cool N-type C stars (Weinberg \& Nikolaev 2001; Demers et al. 2002; Cioni \& Habing 2003; Kang et al. 2006), essentially leading to $(J-K)_{0}>1.4$ (Davidge 2005) and $(H-K)_{0}>0.45$ (Hughes \& Wood 1990) criteria. A critical analysis of these methods is given by Battinelli \& Demers (2007).

We decided to adopt these color limits (regardless of the absorption along the line of sight) along with spatial limits to select disk candidates visible in the Northern Autumn sky, and two additional photometric limits to avoid dust-enshrouded $\mathrm{C}$ stars or objects too faint for the DAO spectrograph, so that our initial constraints are: $1.4<(J-K)<2.3,(H-K)>0.45, K_{\mathrm{s}}<12.0$, $60^{\circ}<\ell<220^{\circ}$ and $3^{\circ}<|b|<5^{\circ}$. The latitude range is set to avoid regions with high and unreliable reddening and to exclude stars too far from the galactic plane.

With these limits we therefore selected from the 2MASS point source catalogue a preliminary list of candidates. Coordinates of each candidate were fed into Schlegel et al. (1998) reddening software to determine the individual reddening and compute the intrinsic colours. Only stars with $(J-K)_{0}>1.4$ and $(H-K)_{0}>0.45$ are retained. To exclude high extinction regions where the Schlegel et al. (1998) results are less reliable we finally set an upper limit of $E(B-V) \approx 0.6(E(J-K) \approx 0.32)$.

\subsection{Distance estimates}

Weinberg \& Nikolaev (2001) have shown that the LMC C stars follow a tight magnitude-color relation in the $(J-K)-K$ plane. Assuming a distance modulus $\mu_{0, \mathrm{LMC}}=18.5$ and an average reddening $E(B-V))=0.2$ (Harris et al. 1997), we transform the Weinberg \& Nikolayev's relation for the LMC into a more general relation based on intrinsic color and magnitude, thus obtaining:

$M_{K}=-6.31-0.99(J-K)_{0}$.

The distance of each star is then computed using its apparent $K$ magnitude, reddening and absolute $K$ magnitude. For observational purposes we also compute the apparent $I$ magnitude for the candidates assuming $M_{I}=-4.6$ (Battinelli \& Demers 2005b).

To determine the galactocentric distances for our candidates we adopt a distance between the Sun and the Galactic center $R_{0}=7.62 \pm 0.32 \mathrm{kpc}$ (Eisenhauer et al. 2005). This value was essentially confirmed by near infrared observations of red clump stars close to the Galactic Center (Nishiyama et al. 2006). The C star candidates are given in Table 1: equatorial coordinates and near-IR photometry are from 2MASS; reddening is from Schlegel et al. (1998); I magnitudes and distances (from the Sun, the Galactic center and the Galactic plane, given in kpc) are estimated as described above. We stress that distances given in Table 1 are obtained under the assumption that the star is a genuine $\mathrm{C}$ star, most of them are indeed included in the General Catalogue of Galactic Carbon stars (Alksnis et al. 2001) but, as we shall see in Sect. 4, some of them are M stars. Figure 1 shows the distribution of the 103 targets projected onto the Galactic plane. The uneven distribution is due to the patchy reddening. This is particularly evident in the Camelopardalis region toward $l=150^{\circ}$.

Among the candidates there are 8 Miras and 1 SRa star of known period, the $P-L$ relation (Feast et al. 2002) can be used to estimate their $M_{K}$ magnitude, independently from the one obtained from Eq. (1). Comparison of these two estimates yields a mean difference of $\left\langle\Delta M_{K}\right\rangle=0.10 \pm 0.15$.

\section{Observations}

Spectra were obtained during six nights in October 2006 with the spectrograph attached to the Cassegrain focus of the Dominion Astrophysical Observatory $1.8 \mathrm{~m}$ Plaskett Telescope. We selected the $2161 \mathrm{R}$ grating giving a dispersion of $30 \AA / \mathrm{mm}$. The SITe2 detector has $15 \mu$ pixels, corresponding to $0.45 \AA /$ pixel. We adopted a slit width of 1 arcsec corresponding to $1.5 \AA$ on the focal plane. The spectral region covered ranges from 6000 to $6900 \AA$. CdNe comparison spectra were taken just before and after each target exposure. Exposures depend on the apparent 


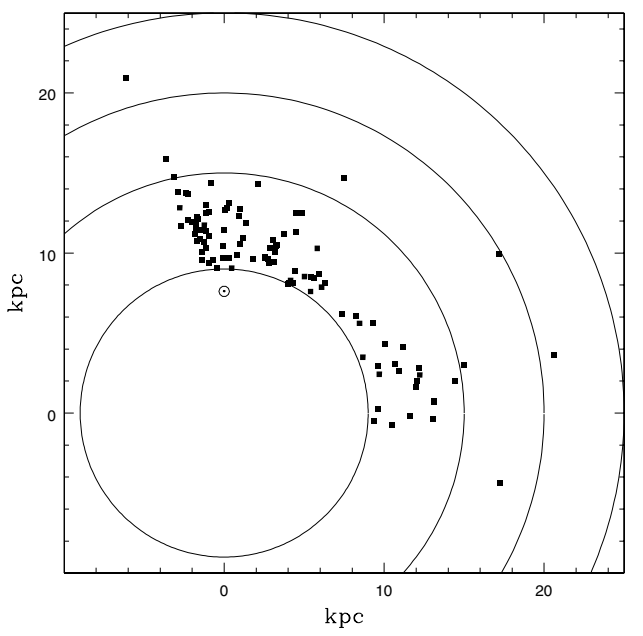

Fig. 1. Distribution of the stars in Table 1 projected onto the Galactic plane. Concentric circles refer to galactocentric distances: 9, 15, 20, $25 \mathrm{kpc}$.

Table 2. Heliocentric velocities of T3 using T22 as template.

\begin{tabular}{cc}
\hline \hline Night & $V_{\odot} \mathrm{km} \mathrm{s}^{-1}$ \\
\hline 4 & $39.2 \pm 7.7$ \\
5 & $47.2 \pm 12.8$ \\
6 & $43.5 \pm 8.3$ \\
\hline
\end{tabular}

magnitude of the targets, they range from 5 to 45 min yielding $\mathrm{S} / \mathrm{N}$ from 30 to 70 .

Two Totten \& Irwin (1998) C stars were used as velocity standards: $2259+1749$ (we shall call T22), with a published heliocentric velocity of $+17 \pm 3 \mathrm{~km} \mathrm{~s}^{-1}$ and $0340+0701$ (we call T3), with a velocity of $+36 \pm 3 \mathrm{~km} \mathrm{~s}^{-1}$. T22 was observed every night while $\mathrm{T} 3$, because of its position in the sky, could be observed only during the last three nights.

Over one hundred spectra were acquired for a total of 89 candidates, 76 of them are confirmed $C$ stars while 13 are not. These latter are mostly $\mathrm{M}$ stars for which our templates are unsuitable to obtain the velocities. Thanks to the good weather conditions during our observing run, 16 stars have been observed on two different nights. In the following sections we discuss results for the $76 \mathrm{C}$ stars, 16 of them having been observed twice. In Fig. 2 it is shown example of spectra. One known C star (No. 2), one not previously classified (No. 28) and two M stars (Nos. 90 and 101) which were not known to be M stars.

\subsection{Velocity determination}

Spectra are analyzed with the relevant IRAF tasks, they are wavelength calibrated with the $\mathrm{CdNe}$ spectra. The wavelength calibrations done with the $\mathrm{CdNe}$ spectra preceding and following the exposure are essentially identical. We could not detect systematic differences in the resulting radial velocities, even for long exposures. For this reason, as a rule we use only one $\mathrm{CdNe}$ spectrum. The radial velocities are obtained with the IRAF task FXCOR which employs a cross-correlation algorithm.

Table 2 presents the radial velocities of T3 obtained in three nights, using the primary standard T22 as template. This table demonstrates that the mean of the derived velocities of T3 is $43 \pm 5 \mathrm{~km} \mathrm{~s}^{-1}$, which is marginally within the error of the published velocity. Rather than averaging the velocities obtained
Table 3. Stars with published radial velocities.

\begin{tabular}{lccccc}
\hline \hline Name & $V_{\mathrm{h}}$ & ref $\dagger$ & Id & $V_{\mathrm{h}}$ & $\Delta V$ \\
\hline WWCas & -59 & $(1)$ & 5 & -62 & +3 \\
DYPer & -46.8 & $(4)$ & 15 & -28 & -19 \\
AVPer & +51 & $(1)$ & 20 & +61 & -10 \\
CGCS918 & -0.1 & $(3)$ & 24 & +10 & -10 \\
CGCS1107 & +12.3 & $(3)$ & 30 & +24 & -12 \\
CGCS1130 & +34.0 & $(3)$ & 32 & +45 & -11 \\
BQAur & +40 & $(2)$ & 33 & +43 & -3 \\
BQAur & +34.7 & $(3)$ & 33 & +43 & -8 \\
- & +30.8 & $(3)$ & 34 & +50 & -19 \\
CGCS1236 & +24 & $(2)$ & 45 & +37 & -13 \\
ITMon & 22.8 & $(5)$ & 58 & 99 & -76 \\
\hline
\end{tabular}

$\dagger$ (1) Sanford (1944); (2) Dean (1976); (3) Metzger \& Schechter (1994); (4) Barnbaum (1992); (5) Walker (1979).

from the two templates we prefer to use only T22 as primary template because it was observed every night. Furthermore, a zero point night-to-night adjustment is found to be unnecessary. Such shifts could be determined by cross correlating T22 of night 4 with its spectra observed on other nights. We find no significant night-to-night difference. Furthermore, the mean difference between the radial velocities of the 16 stars observed twice, is $3.5 \pm 5.5 \mathrm{~km} \mathrm{~s}^{-1}$, well within the expected uncertainty of our cross-correlation. We therefore feel confident that there are no unexpected random errors in the velocities. We stress however that most of our targets are Mira or SR variables that may present pulsating atmospheres. Barnbaum (1992) have shown that radial velocities for such variables present velocity fluctuations from 3 to $9 \mathrm{~km} \mathrm{~s}^{-1}$.

\subsubsection{Zero point adjustment}

Mauron et al. (2004) have observed a $\sim \pm 10 \mathrm{~km} \mathrm{~s}^{-1}$ dispersion in the velocity residuals of several APM stars they used as velocity templates. Such zero point uncertainty suggest that the Totten \& Irwin (1998) velocity determination of C stars may have errors larger than the quoted values. For this reason, our radial velocities, based on a single template, may require a zero point adjustment. We searched the literature finding that 10 of our targets have published velocities (BQ Aur with two independent measurements). In Table 3 we list those stars and compare the published heliocentric velocities to ours.

At first glance we see that IT Mon stands out with its abnormal $\Delta V_{\mathrm{h}}$. Averaging the other 9 measurements we have a $\left\langle\Delta V_{\mathrm{h}}\right\rangle \sim-10 \mathrm{~km} \mathrm{~s}^{-1}$ and $\sigma_{\Delta V_{\mathrm{h}}}=6.6 \mathrm{~km} \mathrm{~s}^{-1}$. It is therefore well justified to exclude IT Mon that is at $\sim 10 \sigma_{\Delta V_{\mathrm{h}}}$ from the computed average. This suggests a zero point offset of $\sim-10 \mathrm{~km} \mathrm{~s}^{-1}$ to be applied to our estimated velocities. It is interesting to note than none of the Aaronson \& Mould (1990) numerous C stars with known velocities is among our targets. The reason is simply that most of their objects are in our exclusion zone $|b|<3^{\circ}$.

\section{Results}

In Table 4 we list the $\mathrm{C}$ star candidate of Table 1 along with their Galactic coordinates, their observed heliocentric velocities (in $\mathrm{km} \mathrm{s}^{-1}$ ) corrected for the zero-point adjustment (see above), the velocity uncertainty as given by FXCOR and the corresponding velocities relative to the LSR. We adopt the solar motion from Dehnen \& Binney (1998). A number of candidates 


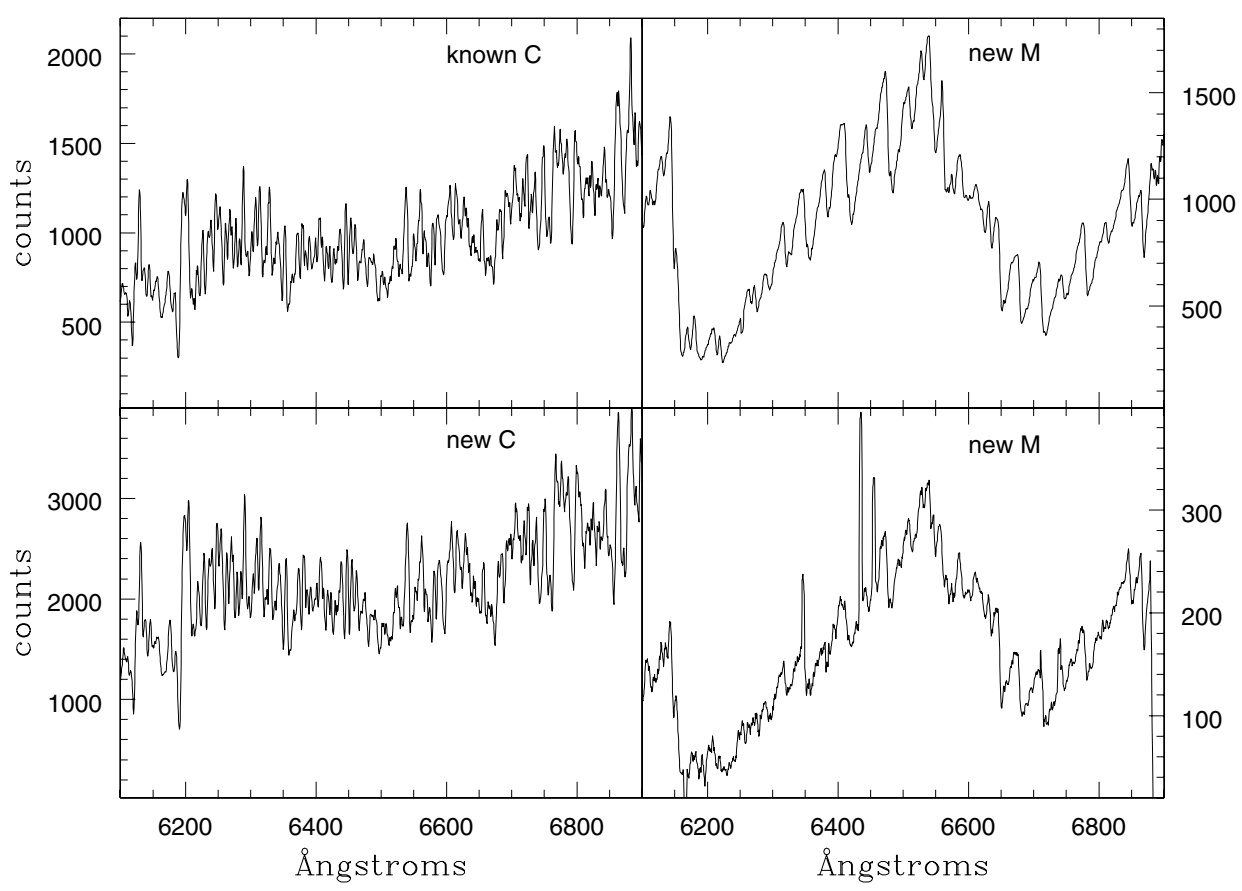

Fig. 2. Examples of spectra of C (left panels) and M stars (right panels). Spectra are smoothed by 9-pixel box car.

Table 4. Radial velocities of $\mathrm{C} \operatorname{stars}^{a}$.

\begin{tabular}{lcccccl}
\hline \hline Id & $\ell$ & $b$ & $V_{\mathrm{h}}$ & err & $V_{\text {LSR }}$ & Notes \\
\hline 1 & 120.8 & -4.8 & -69 & 6 & -70 & V382 Cas \\
2 & 122.3 & -3.2 & -36 & 10 & -37 & CGCS 111 \\
3 & 126.6 & -4.5 & -62 & 5 & -64 & V645 Cas \\
4 & 127.5 & -4.9 & -52 & 2 & -55 & \\
5 & 128.6 & -4.7 & -72 & 8 & -75 & \\
6 & 129.6 & -4.3 & -100 & 2 & -103 & \\
7 & 129.7 & -3.5 & -36 & 10 & -39 & V918 Cas \\
8 & 130.9 & -4.5 & -35 & 10 & -38 & CGCS 282 \\
9 & 131.2 & -4.9 & -82 & 2 & -85 & EW Per \\
10 & 133.1 & -4.5 & -38 & 4 & -42 & CN Per \\
11 & 133.2 & -3.1 & - & - & - & no obs.; CGCS 315 \\
12 & 135.1 & -4.3 & -64 & 10 & -68 & \\
\hline
\end{tabular}

a Table 4 is presented in its entirety in the electronic edition of Astronomy \& Astrophysics. A portion is shown here for guidance regarding its form and content. Velocities are in $\mathrm{km} \mathrm{s}^{-1}$ units.

have spectra indicating their non-C star nature, half of them are $M$ types with colours close to our adopted blue limit. The other spectra show numerous emission lines in the observed region. If emission lines are also present in the NIR, then their colours could be affected. A few stars in Table 4 were not observed due to their faintness. In the last column alternative names for known objects are given along with comments concerning the observations.

In Fig. 3 we compare our $\mathrm{C}$ star radial velocities with two sets available in the literature for the Northern hemisphere, namely: Aaronson et al. (1990) and Metzger \& Schechter (1994) data toward the anticenter. We stress that this plot involves no knowledge of individual stellar distances. From simple geometry, the observed velocity relative to the LSR for stars in the direction $(l, b)$ with galactocentric distance $R$ and rotation velocity $V_{\text {ROT }}$ is given by:

$V_{\mathrm{LSR}}=\left(\frac{R_{0}}{R} \cdot V_{\mathrm{ROT}}-220\right) \sin l \cdot \cos b$

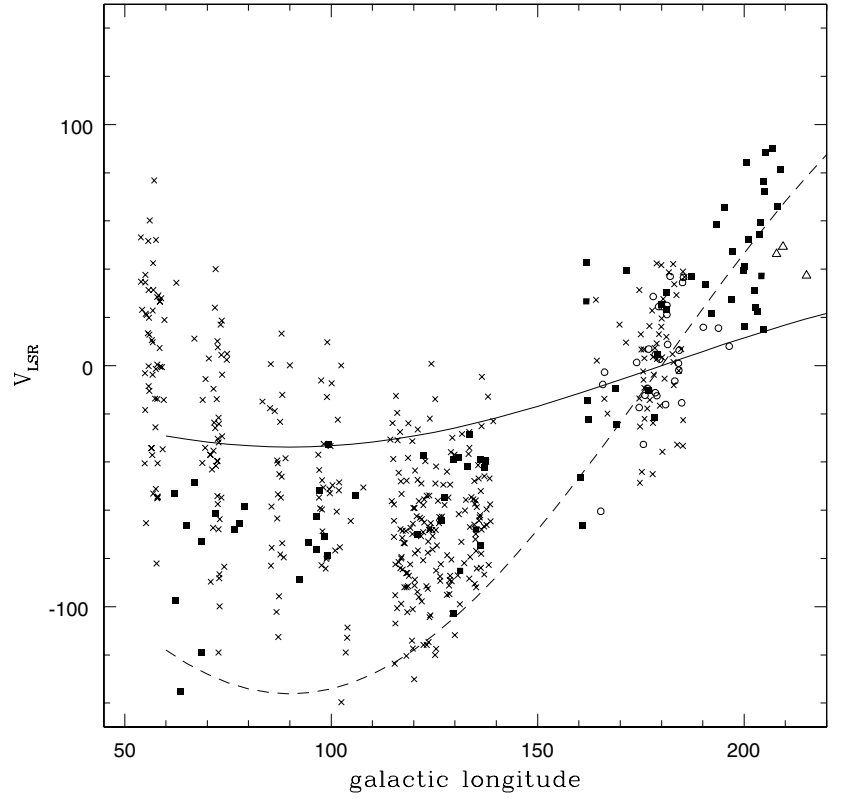

Fig. 3. Radial velocities relative to the LSR: present paper (solid dots), Aaronson et al. 1990 (crosses) and Metzger \& Schechter 1994 (open circles). The two curves correspond to the expected $V_{\mathrm{LSR}}$ at galactocentric distances of $9 \mathrm{kpc}$ (solid) and $20 \mathrm{kpc}$ (dashed) with the assumption of a flat $\left(220 \mathrm{~km} \mathrm{~s}^{-1}\right)$ rotation curve beyond the Sun.

where we adopt $220 \mathrm{~km} \mathrm{~s}^{-1}$ for the LSR motion (Kerr \& LyndenBell 1986). The two curves in Fig. 3 are obtained from the above relation with with $b=0$ and assuming a flat rotation beyond the solar circle. In Fig. 3 all the Aaronson et al. stars with positive velocities and $l<90^{\circ}$ are located inside the solar circle. Most of our stars have $V_{\text {LSR }}$ compatible with the galactocentric distance range shown in Fig. 1. Towards the anti-center, the mean velocity is expected to be zero if the LSR has no radial motion. There are 68 stars with $175^{\circ}<l<185^{\circ}$ with $\left\langle V_{\mathrm{LSR}}\right\rangle=2.6 \mathrm{~km} \mathrm{~s}^{-1}$ and a dispersion $\pm 20 \mathrm{~km} \mathrm{~s}^{-1}$ typical for intermediate-age population 


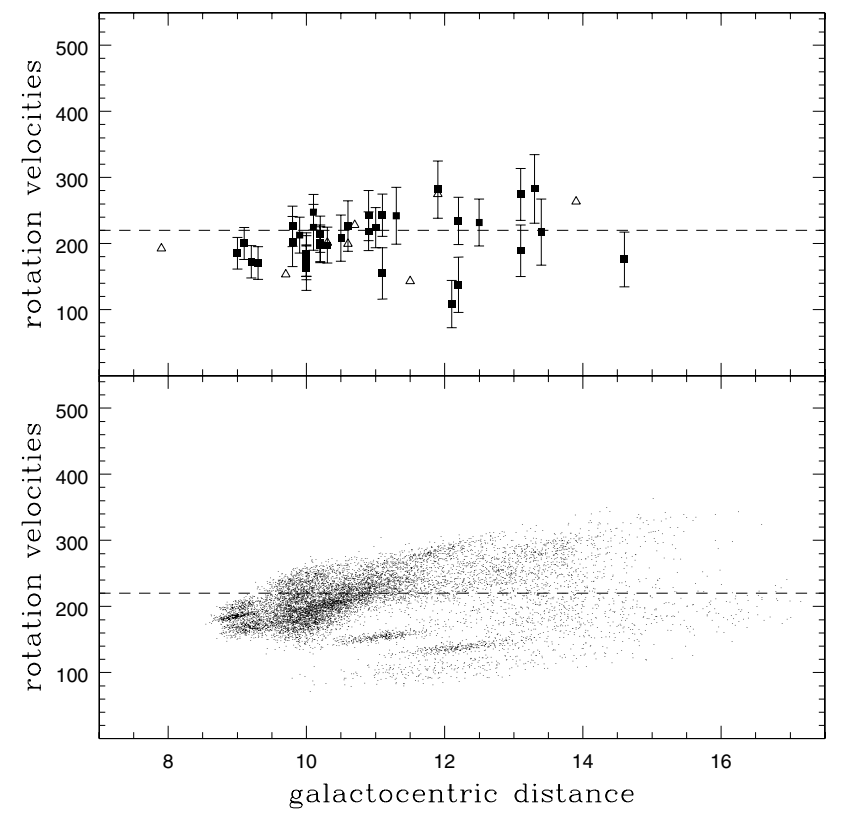

Fig. 4. Upper panel: rotation velocities as a function of distances in kpc. The open triangles are data from Aaronson et al. (1990). Stars $60^{\circ}<\ell<$ $150^{\circ}$ are plotted. Bottom panel: result of a Monte Carlo simulation of the rotation velocities taking into account the errors attached to each data point (see Sect. 5.1.1).

(Feast et al. 2006). It is however puzzling to note that a few stars have observed $V_{\mathrm{LSR}}$ too high for their longitude, to belong to the thin disk. We shall come back to those stars, located at $\ell>160^{\circ}$, in the next section.

\section{Discussion}

To calculate the rotation velocity, assuming circular motion, we can simply inverse Eq. (2), leading to:

$V_{\mathrm{ROT}}=\frac{R}{R_{0}}\left(\frac{V_{\mathrm{LSR}}}{\sin l \cdot \cos b}-220\right)$,

because of the $\sin (l)$ at the denominator, $V_{\mathrm{ROT}}$ is not well defined when the longitude is close to $180^{\circ}$. For this reason we divide the discussion into two longitude zones.

\subsection{Longitudes less than $150^{\circ}$}

The rotation velocities of $\mathrm{C}$ stars are plotted, as a function of the galactocentric distances in Fig. 4 (upper panel), for $60^{\circ}<$ $\ell<150^{\circ}$. The error bars on $V_{\text {ROT }}$ take into account a $\pm 20 \mathrm{~km} \mathrm{~s}^{-1}$ random motion and a $10 \%$ uncertainty in the distance determination. The open triangles, in Fig. 4, are C stars from Aaronson et al. (1990). Very few of their C stars satisfy our acceptance criteria. This figure suggests that the rotation curve of the Galaxy is essentially flat at least up to $15 \mathrm{kpc}$ from the Galactic center.

This, indeed can be better seen if we plot the mean velocities. We bin the 35 stars into $1 \mathrm{kpc}$ wide bins. These mean velocities are shown in Fig. 5, where the error bars correspond to the standard deviation of each mean. This result essentially confirms the rotation curve derived from planetary nebulae by Maciel \& Lago (2005) while the comparison with the conclusions of Amaral et al. (1996) is more difficult because of their adoption of different values of $R_{0}$ and for the LSR circular velocity. In any case, we feel that the small number of points extending to $14 \mathrm{kpc}$ does

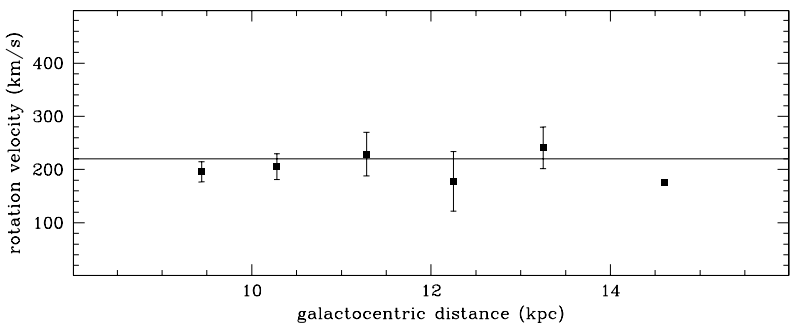

Fig. 5. Mean rotation velocity of the stars plotted in Fig. 4 (upper panel) grouped into bins of seven.

not warrant a more detailed analysis. We hope to extend this curve in the near future.

\subsubsection{Combined distance and $V_{\mathrm{h}}$ uncertainties}

In Fig. 4, for clarity reasons, only errorbars in the computed rotation velocities are displayed. It is however interesting to estimate the combined effect of the uncertainties in the heliocentric distances and velocities on the derived rotation velocities. We performed a Monte Carlo test assuming: i) Gaussian distributions of errors; $i$ ) errors given in Table 4 as individual heliocentric velocity dispersion; iii) a 0.2 mag dispersion in the colour - absolute $K$ magnitude given in Eq. (1); vi) negligible errors in the individual reddening and apparent $K$ magnitude. For each star we generated 300 simulated data points under the above assumptions. In the bottom panel of Fig. 4 the location of all these simulated data points reveals the real uncertainty of the rotation curve.

\subsection{Longitudes from $150^{\circ}$ to $220^{\circ}$}

Our radial velocity data in regions closer to the anticenter is more complicated to interpret, as Fig. 6 demonstrates. The scatter of the calculated $V_{\text {ROT }}$ and and their error bars are much larger than seen in Fig. 4. The two dotted lines limit the region occupied by the velocities of Fig. 4. Outside this region we note, in Fig. 6, two groups of stars: two relatively nearby stars with very high velocities and ten stars with $V_{\text {ROT }}$ significantly less than $220 \mathrm{~km} \mathrm{~s}^{-1}$. The two stars are stars No. 20 and No. 23. A search of SIMBAD database revealed that the first one is AV Per a SRB-type variable of unknown period. Its radial velocity obtained by Sanford (1944) confirms our measures, see Table 3. The second star is a known C star listed as CGCS 873 but nothing else is known about it. Both stars have unusually large $V_{\mathrm{LSR}}$ for their longitude, they standout in Fig. 3 at $\ell \sim 161^{\circ}$. Even though the two stars are only 8 degrees apart in the sky, they are some $1400 \mathrm{pc}$ from each other. We tentatively conclude that these two stars do not belong neither to the thin nor the thick disk.

The ten stars, with $V_{\text {ROT }}$ significantly less than $220 \mathrm{~km} \mathrm{~s}^{-1}$, do not have abnormally large distances from the plane. One of them is IT Mon that we already discussed in Sect. 3.1.1. No previous measurements are available for the other stars of the group. The stars of the group also stand out in Fig. 3, they are near $\ell \sim 200^{\circ}$. They could be members of the thick disk that is known to lags the thin disk by some $50 \mathrm{~km} \mathrm{~s}^{-1}$ (Soubiran et al. 2003). The ratio of thick to thin disk stars $z \sim 800 \mathrm{pc}$ is not negligible. An accurate ratio is difficult to determined due to the ill-defined thick disk parameters, see Norris (1999) for a review. It would be, however, quite unusual for such stars to show up only the the longitude zone where the $V_{\mathrm{ROT}}$ are less accurately determined. 


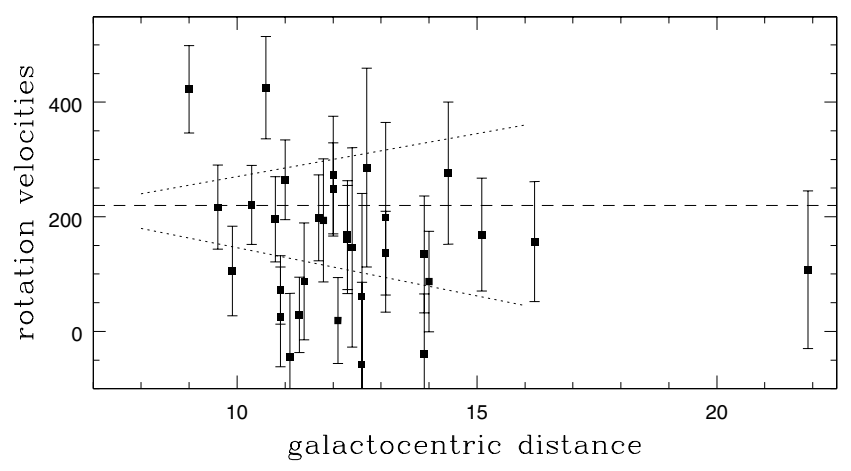

Fig. 6. Rotation velocities as a function of distances in $\mathrm{kpc}$. Stars in the zones $150^{\circ}<\ell<170^{\circ}$ and $190^{\circ}, \ell<220^{\circ}$ are plotted. The dotted lines limit the region occupied by the points of Fig. 4.

We believe that there is a more logical explanation for the high $V_{\mathrm{LSR}}$ stars seen at $\ell \sim 200^{\circ}$. They are members of the Canis Major overdensity kinematically characterized by Martin et al. (2004). Indeed the bulk of this density enhancement is at $7 \mathrm{kpc}$ from the Sun, close enough to include members within our distance range. It is known that the Sagittarius Stream crosses the Galactic plane in the direction of the anti center, however, simulations (Bellazzini private communication) show that its members are then more than $30 \mathrm{kpc}$ from the Galactic center, too far to be include in our survey.

\section{Conclusions}

The two farthest stars in Fig. 6 (No. 49 and No. 54) have rotation velocities less than $220 \mathrm{~km} \mathrm{~s}^{-1}$ which could suggest that the rotation curve of the Galaxy is declining. Unfortunately these two stars have Galactic longitudes of $204^{\circ}$ and $208^{\circ}$ and are most probably associated with the Canis Major structure. Observations of fainter, thus more distant, $\mathrm{C}$ stars must be limited, at least from the Northern Hemisphere, to longitudes less than $150^{\circ}$ to avoid the known streams. We hope to complete such survey in the near future.

\footnotetext{
Acknowledgements. The authors would like to thank Dr. Dmitry Monin for his valuable advice that helped us to achieve a successful run. This research is funded by the Natural Sciences and Engineering Research Council of Canada. This publication makes use of data products from the Two Micron All Sky Survey, which is a joint project of the University of Massachusetts and the Infrared Processing and Analysis Center/California Institute of Technology, funded by the National Aeronautics and Space Administration and the National Science Foundation.
}

Note added in proof. In August 2007 we obtained an independent estimate of the radial velocity of T22, used as primary standard in this paper. Its heliocentric velocity was found to be $5 \pm 4 \mathrm{~km} \mathrm{~s}^{-1}$, i.e. about $10 \mathrm{~km} \mathrm{~s}^{-1}$ smaller that the published by Totten \& Irwin. This discrepancy fully explains the introduction of a zero point adjustement adopted in this paper.

\section{References}

Aaronson, M., Blanco, V. M., Cook, K. H., \& Schechter, P. L. 1989, ApJS, 70, 637

Aaronson, M., Blanco, V. M., Cook, K. H., Olszewski, E. W., \& Schechter, P. L. 1990, ApJS, 73, 841

Alksnis, A., Balklovs, A., Dzervitis, U., et al. 2001, Bal. Astron., 10, 1

Amaral, L. H., Ortiz, R., Lépine, J. R. D., \& Maciel, W. J. 1996, MNRAS, 281, 339

Barnbaum, C. 1992, AJ, 104, 1585

Battinelli, P., \& Demers, S. 2005a, A\&A, 434, 657

Battinelli, P., \& Demers, S. 2005b, A\&A, 442, 159

Battinelli, P., \& Demers, S. 2007, A\&A, in press

Carignan, C., Chemin, L., Hutchmeir, M. K., \& Lockman, F. J. 2006, ApJ, 641, L109

Cioni, M.-R. L., \& Habing, H. J. 2003, A\&A, 402, 133

Norris, J. E. 1999, A\&SS, 265, 213

Davidge, T. J. 2005, AJ, 130, 2087

Dean, C. A. 1976, AJ, 81,364

Dehnen, W., \& Binney, J. 1998, MNRAS, 293, 429

Demers, S., Battinelli, P., \& Dallaire, M. 2002, AJ, 123, 3428

Eisenhauer, F., Genzel, R., Alexander, T., et al. 2005, ApJ, 628, 246

Feast, M., Whitelock, P., \& Menzies, J. 2002, MNRAS, 329, L7

Feast, M., Whitelock, P., \& Menzies, J. 2006, MNRAS, 369, 791

Frinchaboy, P. M., \& Majewski, S. R. 2005, in Island Universes: structure and Evolution of Disk Galaxies, ed. R. de Jong (Dortrecht: Springer), in press [arXiv:astro-ph/0508666]

Harris, J., Zaritsky, D., \& Thompson, I. 1997, AJ, 114, 1933

Hughes, S. M. G., \& Wood, P. R. 1990, AJ, 99, 784

Kang, A., Sohn, Y.-J., Kim, H.-I., et al. 2006, A\&A, 454, 717

Kerr, E. J., \& Lynden-Bell, D. 1986, MNRAS, 221, 1023

Maciel, W. J., \& Lago, L. G. 2005, Rev. Mex. A\&A, 41, 383

Martin, N. F., Ibata, R. A., Conn, B. C., et al. 2004, MNRAS, 355, L33

Mauron, N., Azzopardi, M., Gigoyan, K., \& Kendall, T. R. 2004, A\&A, 418, 77

Merrifield, M. R. 1992, AJ, 103, 1552

Metzger, M. R., \& Schechter, P. L. 1994, ApJ, 420, 177

Nakashima, J., Jiang, B. W., Deguchi, S., Sadakane, K., \& Nakada, Y. 2000, PASJ, 52, 275

Nishiyama, S., Nagata, T., Sato, S., et al. 2006, ApJ, 647, 1093

Sanford, R. F. 1944, ApJ, 99, 145

Schechter, P. L., Aaronson, M., Cook, K. H., \& Blanco, V. M. 1988, in The Outer Galaxy, Lecture Notes in Physics, 306, ed. L. Blitz, \& F. J. Lockman (Berlin: Springer-Verlag), 31

Schlegel, D., Finkbeiner, D., \& Davis, M. 1998, ApJ, 500, 525

Sneider, S. E., \& Terzian, Y. 1983, ApJ, 274, L61

Soubiran, C., Bienaymé, O., \& Siebert, A. 2003, A\&A, 398, 141

Totten, E. J., \& Irwin, M. J. 1998, MNRAS, 294, 1

Walker, A. R. 1979, SAAO Circulars 1, 192

Weinberg, M. D., \& Nokolaev, S. 2001, ApJ, 548, 712 


\section{Online Material}


Table 1. C star candidates.

\begin{tabular}{|c|c|c|c|c|c|c|c|c|c|c|}
\hline Id & \multicolumn{2}{|c|}{ RA J2000 Dec } & $K_{s}$ & $\left(J-K_{s}\right)$ & $\left(H-K_{s}\right)$ & $E_{(B-V)}$ & $I$ & $d_{\odot}$ & $d_{G C}$ & $z$ \\
\hline 1 & 03537.5 & $5800 \quad 24.3$ & 4.93 & 1.95 & 0.68 & 0.43 & 9.02 & 3.6 & 10.0 & -0.30 \\
\hline 2 & $\begin{array}{lll}0 & 46 & 17.9\end{array}$ & $\begin{array}{lll}59 & 37 & 40.1\end{array}$ & 4.91 & 1.89 & 0.62 & 0.58 & 9.10 & 3.3 & 9.8 & -0.19 \\
\hline 3 & $\begin{array}{lll}1 & 18 & 52.8\end{array}$ & 580931.0 & 4.68 & 2.10 & 0.73 & 0.49 & 8.99 & 3.4 & 10.0 & -0.26 \\
\hline 4 & $\begin{array}{lll}1 & 25 & 18.9\end{array}$ & $\begin{array}{lll}57 & 39 & 18.0\end{array}$ & 5.19 & 2.01 & 0.65 & 0.56 & 9.48 & 4.0 & 10.5 & -0.34 \\
\hline 5 & $134 \quad 16.1$ & $\begin{array}{lll}57 & 43 & 27.6\end{array}$ & 5.00 & 1.80 & 0.60 & 0.59 & 9.11 & 3.3 & 10.0 & -0.27 \\
\hline 6 & $\begin{array}{lll}1 & 42 & 08.7\end{array}$ & $\begin{array}{lll}57 & 55 & 32.1\end{array}$ & 6.25 & 1.73 & 0.57 & 0.51 & 10.20 & 5.8 & 12.2 & -0.43 \\
\hline 7 & 14358.3 & 584105.0 & 4.89 & 1.92 & 0.69 & 0.56 & 9.09 & 3.3 & 10.1 & -0.20 \\
\hline 8 & 15055.6 & $\begin{array}{lll}57 & 28 & 51.0\end{array}$ & 5.54 & 1.62 & 0.54 & 0.32 & 9.19 & 4.3 & 10.9 & -0.34 \\
\hline 9 & 15215.5 & 565802.5 & 5.54 & 1.66 & 0.59 & 0.30 & 9.21 & 4.4 & 11.1 & -0.38 \\
\hline 10 & 20639.6 & 565106.2 & 4.89 & 2.13 & 0.85 & 0.43 & 9.16 & 3.9 & 10.6 & -0.30 \\
\hline 11 & $\begin{array}{lll}2 & 10 & 16.1\end{array}$ & $\begin{array}{lll}58 & 14 & 27.0\end{array}$ & 7.32 & 2.01 & 0.68 & 0.62 & 11.67 & 10.3 & 16.5 & -0.55 \\
\hline 12 & 22057.6 & $\begin{array}{lll}56 & 23 & 20.1\end{array}$ & 6.19 & 2.14 & 0.81 & 0.49 & 10.54 & 6.9 & 13.4 & -0.52 \\
\hline 13 & $\begin{array}{lll}2 & 29 & 39.7\end{array}$ & $\begin{array}{lll}56 & 11 & 30.2\end{array}$ & 5.50 & 1.84 & 0.62 & 0.45 & 9.50 & 4.4 & 11.3 & -0.32 \\
\hline 14 & 23246.3 & $\begin{array}{lll}56 & 29 & 27.0\end{array}$ & 5.99 & 1.74 & 0.65 & 0.52 & 9.97 & 5.2 & 11.9 & -0.33 \\
\hline 15 & 23517.1 & $5608 \quad 44.7$ & 4.41 & 1.96 & 0.78 & 0.55 & 8.64 & 2.7 & 9.8 & -0.18 \\
\hline 16 & 23808.6 & $56 \quad 2104.2$ & 6.09 & 2.28 & 0.80 & 0.60 & 10.68 & 6.6 & 13.3 & -0.41 \\
\hline 17 & 43001.3 & $\begin{array}{lll}41 & 50 & 27.7\end{array}$ & 3.96 & 2.13 & 0.82 & 0.56 & 8.37 & 2.4 & 9.9 & -0.19 \\
\hline 18 & $430 \quad 55.8$ & $\begin{array}{lll}41 & 34 & 15.1\end{array}$ & 4.92 & 2.01 & 0.70 & 0.59 & 9.24 & 3.5 & 10.9 & -0.28 \\
\hline 19 & $\begin{array}{lll}4 & 37 & 17.1\end{array}$ & $40 \quad 4658.8$ & 6.35 & 2.10 & 0.72 & 0.59 & 10.76 & 7.0 & 14.4 & -0.52 \\
\hline 20 & $\begin{array}{lll}4 & 39 & 35.3\end{array}$ & $\begin{array}{lll}41 & 37 & 11.3\end{array}$ & 3.04 & 1.96 & 0.75 & 0.52 & 7.23 & 1.5 & 9.0 & -0.09 \\
\hline 21 & $\begin{array}{lll}4 & 40 & 38.4\end{array}$ & $\begin{array}{lll}41 & 29 & 52.4\end{array}$ & 5.41 & 2.12 & 0.77 & 0.61 & 9.85 & 4.5 & 12.0 & -0.26 \\
\hline 22 & $\begin{array}{lll}5 & 10 & 27.7\end{array}$ & $\begin{array}{lll}33 & 59 & 38.3\end{array}$ & 3.90 & 1.91 & 0.77 & 0.58 & 8.11 & 2.1 & 9.7 & -0.12 \\
\hline 23 & $\begin{array}{lll}5 & 11 & 38.2\end{array}$ & $46 \quad 1704.3$ & 4.80 & 1.93 & 0.66 & 0.62 & 9.07 & 3.1 & 10.6 & 0.22 \\
\hline 24 & 52300.2 & 292416.8 & 6.11 & 1.77 & 0.58 & 0.54 & 10.14 & 5.5 & 13.1 & -0.37 \\
\hline 25 & $\begin{array}{lll}5 & 33 & 37.8\end{array}$ & $\begin{array}{lll}40 & 28 & 30.2\end{array}$ & 5.86 & 1.90 & 0.66 & 0.54 & 10.03 & 5.2 & 12.7 & 0.37 \\
\hline 26 & $\begin{array}{lll}5 & 34 & 42.4\end{array}$ & $\begin{array}{lll}40 & 15 & 38.0\end{array}$ & 5.49 & 2.07 & 0.81 & 0.50 & 9.78 & 4.8 & 12.4 & 0.34 \\
\hline 27 & 55105.5 & $\begin{array}{lll}17 & 22 & 17.1\end{array}$ & 5.70 & 1.85 & 0.64 & 0.37 & 9.63 & 5.0 & 12.6 & -0.43 \\
\hline 28 & $\begin{array}{lll}5 & 56 & 38.5\end{array}$ & $\begin{array}{lll}14 & 57 & 29.0\end{array}$ & 5.63 & 1.89 & 0.68 & 0.35 & 9.58 & 5.0 & 12.6 & -0.43 \\
\hline 29 & 55646.3 & $16 \quad 32 \quad 31.2$ & 5.97 & 1.87 & 0.66 & 0.48 & 10.03 & 5.5 & 13.1 & -0.40 \\
\hline 30 & $\begin{array}{lll}5 & 56 & 48.4\end{array}$ & 305009.0 & 5.57 & 2.14 & 0.80 & 0.53 & 9.96 & 5.1 & 12.7 & 0.27 \\
\hline 31 & $\begin{array}{lll}5 & 57 & 14.8\end{array}$ & $\begin{array}{lll}32 & 22 & 39.1\end{array}$ & 5.96 & 1.77 & 0.66 & 0.48 & 9.93 & 5.2 & 12.9 & 0.36 \\
\hline 32 & 60052.2 & $\begin{array}{lll}30 & 45 & 25.4\end{array}$ & 5.17 & 1.98 & 0.72 & 0.62 & 9.49 & 3.8 & 11.4 & 0.25 \\
\hline 33 & 60143.7 & $2927 \quad 16.7$ & 3.95 & 1.85 & 0.75 & 0.48 & 8.00 & 2.1 & 9.8 & 0.12 \\
\hline 34 & 60245.9 & $2938 \quad 44.6$ & 4.73 & 1.67 & 0.60 & 0.49 & 8.61 & 2.8 & 10.4 & 0.18 \\
\hline 35 & 60605.8 & $\begin{array}{lll}11 & 57 & 37.4\end{array}$ & 4.97 & 2.07 & 0.74 & 0.44 & 9.19 & 3.9 & 11.4 & -0.30 \\
\hline 36 & 61051.5 & $\begin{array}{lll}09 & 22 & 35.0\end{array}$ & 5.55 & 1.98 & 0.72 & 0.41 & 9.65 & 4.9 & 12.3 & -0.39 \\
\hline 37 & $\begin{array}{lll}6 & 12 & 17.5\end{array}$ & 092101.9 & 5.48 & 2.05 & 0.78 & 0.45 & 9.70 & 4.8 & 12.3 & -0.36 \\
\hline 38 & $\begin{array}{lll}6 & 14 & 53.9\end{array}$ & $\begin{array}{lll}08 & 39 & 58.7\end{array}$ & 6.36 & 1.81 & 0.63 & 0.43 & 10.32 & 6.6 & 13.9 & -0.46 \\
\hline 39 & $\begin{array}{lll}6 & 15 & 27.0\end{array}$ & 070556.1 & 4.97 & 1.84 & .65 & .55 & 9.08 & 3.3 & 10.8 & -0.27 \\
\hline 40 & 61606.4 & $\begin{array}{lll}06 & 46 & 37.1\end{array}$ & 5.36 & 2.02 & 0.74 & 40 & 9.49 & 6 & 12.0 & -0.38 \\
\hline 41 & $\begin{array}{lll}6 & 16 & 21.7\end{array}$ & $\begin{array}{lll}09 & 53 & 54.3\end{array}$ & 3.79 & 2.05 & 0.84 & 0.55 & 8.10 & 2.1 & 9.6 & -0.12 \\
\hline 42 & $\begin{array}{llll}6 & 17 & 37.3\end{array}$ & $0937 \quad 35.1$ & 6.15 & 2.04 & 0.75 & 0.46 & 10.36 & 6.5 & 13.9 & -0.34 \\
\hline 43 & $\begin{array}{lll}6 & 18 & 57.2\end{array}$ & $\begin{array}{lll}08 & 12 & 03.0\end{array}$ & 5.30 & 1.91 & 0.79 & 0.48 & 9.41 & 4.1 & 11.6 & -0.25 \\
\hline 44 & $\begin{array}{lll}6 & 19 & 37.2\end{array}$ & $06 \quad 48 \quad 38.5$ & 4.24 & 2.13 & 0.80 & 0.43 & 8.52 & 2.9 & 10.3 & -0.20 \\
\hline 45 & $\begin{array}{lll}6 & 19 & 50.4\end{array}$ & $\begin{array}{lll}05 & 19 & 34.5\end{array}$ & 5.02 & 1.84 & 0.63 & 0.40 & 8.98 & 3.6 & 11.0 & -0.29 \\
\hline 46 & $\begin{array}{lll}6 & 20 & 17.2\end{array}$ & $2504 \quad 34.2$ & 6.36 & 2.03 & 0.72 & 0.60 & 10.71 & 6.8 & 14.4 & 0.57 \\
\hline 47 & $\begin{array}{lll}6 & 20 & 39.8\end{array}$ & $05 \quad 5258.4$ & 5.47 & 1.87 & 0.66 & 0.53 & 9.60 & 4.3 & 11.7 & -0.31 \\
\hline 48 & $\begin{array}{lll}6 & 21 & 53.9\end{array}$ & 062627.1 & 6.67 & 1.90 & 0.64 & 0.46 & 10.75 & 7.8 & 15.1 & -0.49 \\
\hline 49 & $\begin{array}{lll}6 & 23 & 53.4\end{array}$ & $\begin{array}{lll}06 & 27 & 41.9\end{array}$ & 7.07 & 1.92 & 0.69 & 0.59 & 11.30 & 9.0 & 16.2 & -0.49 \\
\hline 50 & $\begin{array}{lll}6 & 29 & 35.2\end{array}$ & $03 \quad 4948.6$ & 5.88 & 1.78 & 0.56 & 0.49 & 9.87 & 5.0 & 12.3 & -0.27 \\
\hline 51 & 63354.1 & 155507.6 & 5.53 & 1.85 & 0.64 & 0.59 & 9.69 & 4.3 & 11.8 & 0.25 \\
\hline 52 & $\begin{array}{lll}6 & 34 & 46.4\end{array}$ & $\begin{array}{lll}17 & 46 & 37.2\end{array}$ & 5.00 & 1.95 & 0.73 & 0.54 & 9.21 & 3.6 & 11.1 & 0.28 \\
\hline 53 & $\begin{array}{lll}6 & 37 & 29.9\end{array}$ & $\begin{array}{lll}14 & 42 & 13.6\end{array}$ & 2.96 & 2.00 & 0.74 & 0.44 & 7.11 & 1.5 & 9.0 & 0.09 \\
\hline 54 & 65259.6 & $\begin{array}{lll}09 & 16 & 37.5\end{array}$ & 7.84 & 1.92 & 0.75 & 0.24 & 11.71 & 14.7 & 21.9 & 1.17 \\
\hline 55 & 65321.3 & $05 \quad 4136.1$ & 6.03 & 1.88 & 0.67 & 0.38 & 10.01 & 5.9 & 13.1 & 0.31 \\
\hline 56 & $\begin{array}{lll}6 & 53 & 32.7\end{array}$ & $\begin{array}{lll}07 & 10 & 32.7\end{array}$ & 5.39 & 1.55 & 0.52 & 0.27 & 8.92 & 4.0 & 11.3 & 0.26 \\
\hline 57 & 65356.9 & $0921 \quad 10.6$ & 6.27 & 1.83 & 0.62 & 0.27 & 10.07 & 6.8 & 14.0 & 0.57 \\
\hline 58 & 65400.1 & 090006.9 & 5.64 & 1.64 & 0.55 & 0.22 & 9.21 & 4.8 & 12.1 & 0.39 \\
\hline 59 & 65704.0 & $06 \quad 1900.6$ & 3.72 & 1.77 & 0.63 & 0.28 & 7.48 & 2.0 & 9.5 & 0.15 \\
\hline 60 & 65707.9 & $\begin{array}{llll}05 & 28 & 51.8\end{array}$ & 5.08 & 1.78 & 0.60 & 0.39 & 8.97 & 3.6 & 10.9 & 0.24 \\
\hline
\end{tabular}


S. Demers and P. Battinelli: Galactic C star kinematics, Online Material p 3

Table 1. continued.

\begin{tabular}{|c|c|c|c|c|c|c|c|c|c|c|}
\hline Id & \multicolumn{2}{|c|}{ RA J2000 Dec } & $K_{s}$ & $\left(J-K_{s}\right)$ & $\left(H-K_{s}\right)$ & $E_{(B-V)}$ & $I$ & $d_{\odot}$ & $d_{G C}$ & $z$ \\
\hline 61 & $700 \quad 29.1$ & $\begin{array}{lll}05 & 11 & 49.5\end{array}$ & 4.50 & 1.72 & 0.63 & 0.29 & 8.22 & 2.8 & 10.2 & 0.22 \\
\hline 62 & $707 \quad 12.7$ & $\begin{array}{lll}01 & 52 & 38.0\end{array}$ & 5.80 & 1.57 & 0.53 & 0.26 & 9.34 & 4.9 & 12.0 & 0.37 \\
\hline 63 & $\begin{array}{lll}7 & 11 & 38.9\end{array}$ & $\begin{array}{lll}00 & 09 & 44.7\end{array}$ & 4.03 & 1.87 & 0.55 & 0.28 & 7.88 & 2.4 & 9.7 & 0.20 \\
\hline 64 & $\begin{array}{lll}19 & 28 & 40.4\end{array}$ & $27 \quad 51 \quad 17.1$ & 7.30 & 2.07 & 0.82 & 0.50 & 11.57 & 11.0 & 10.0 & 0.95 \\
\hline 65 & $1932 \quad 20.6$ & $\begin{array}{lll}27 & 46 & 21.1\end{array}$ & 8.36 & 1.74 & 0.60 & 0.62 & 12.45 & 14.8 & 13.1 & 1.07 \\
\hline 66 & $\begin{array}{lll}19 & 32 & 41.0\end{array}$ & 28601.1 & 8.16 & 1.83 & 0.61 & 0.48 & 12.19 & 14.8 & 13.1 & 1.10 \\
\hline 67 & $1933 \quad 55.3$ & $12 \quad 40 \quad 40.5$ & 7.92 & 1.78 & 0.61 & 0.58 & 12.00 & 12.4 & 9.4 & -0.75 \\
\hline 68 & $\begin{array}{lll}19 & 35 & 18.4\end{array}$ & $\begin{array}{lll}28 & 51 & 10.6\end{array}$ & 7.97 & 1.76 & 0.61 & 0.43 & 11.87 & 13.4 & 12.1 & 0.96 \\
\hline 69 & $1935 \quad 55.1$ & 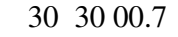 & 7.80 & 1.98 & 0.71 & 0.51 & 12.01 & 13.3 & 12.2 & 1.11 \\
\hline 70 & $1937 \quad 15.6$ & $\begin{array}{lll}31 & 05 & 13.4\end{array}$ & 7.50 & 1.93 & 0.81 & 0.37 & 11.51 & 12.0 & 11.2 & 1.01 \\
\hline 71 & 193805.3 & $29 \quad 10 \quad 57.7$ & 7.47 & 1.90 & 0.71 & 0.59 & 11.68 & 10.7 & 10.0 & 0.70 \\
\hline 72 & $1938 \quad 41.2$ & $29 \quad 45 \quad 15.6$ & 6.98 & 2.05 & 0.77 & 0.46 & 11.21 & 9.6 & 9.3 & 0.65 \\
\hline 73 & $1941 \quad 57.2$ & $\begin{array}{lll}15 & 25 & 13.3\end{array}$ & 7.91 & 1.71 & 0.60 & 0.56 & 11.90 & 12.1 & 9.6 & -0.80 \\
\hline 74 & $\begin{array}{lll}19 & 42 & 38.9\end{array}$ & $\begin{array}{lll}14 & 11 & 36.4\end{array}$ & 8.06 & 1.66 & 0.70 & 0.43 & 11.86 & 13.4 & 10.5 & -1.06 \\
\hline 75 & $\begin{array}{lll}19 & 43 & 13.5\end{array}$ & 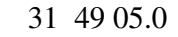 & 7.80 & 2.03 & 0.76 & 0.57 & 12.12 & 13.3 & 12.5 & 0.94 \\
\hline 76 & $\begin{array}{lll}19 & 43 & 21.1\end{array}$ & $\begin{array}{lll}31 & 50 & 40.4\end{array}$ & 7.70 & 1.81 & 0.61 & 0.56 & 11.79 & 11.6 & 11.1 & 0.82 \\
\hline 77 & $1944 \quad 45.5$ & $\begin{array}{lll}17 & 42 & 54.9\end{array}$ & 8.60 & 2.27 & 0.94 & 0.62 & 13.21 & 21.0 & 17.7 & -1.19 \\
\hline 78 & $\begin{array}{lll}19 & 45 & 33.2\end{array}$ & $33 \quad 3108.8$ & 7.83 & 1.81 & 0.61 & 0.39 & 11.74 & 13.1 & 12.5 & 1.02 \\
\hline 79 & $\begin{array}{lll}19 & 47 & 17.5\end{array}$ & $33 \quad 3004.8$ & 8.20 & 1.90 & 0.66 & 0.50 & 12.31 & 15.5 & 14.6 & 1.12 \\
\hline 80 & $1948 \quad 45.6$ & $\begin{array}{lll}18 & 52 & 17.4\end{array}$ & 8.18 & 1.75 & 0.59 & 0.56 & 12.21 & 14.0 & 11.6 & -0.85 \\
\hline 81 & 195405.4 & $2041 \quad 26.3$ & 7.87 & 2.29 & 0.86 & 0.59 & 12.47 & 15.3 & 13.0 & -0.97 \\
\hline 82 & 195606.5 & 360632.8 & 7.44 & 1.92 & 0.64 & 0.58 & 11.66 & 10.6 & 11.0 & 0.73 \\
\hline 83 & 195806.4 & $\begin{array}{lll}36 & 59 & 10.3\end{array}$ & 8.46 & 1.68 & 0.66 & 0.51 & 12.36 & 15.7 & 15.3 & 1.11 \\
\hline 84 & $\begin{array}{lll}19 & 58 & 32.2\end{array}$ & $\begin{array}{lll}36 & 49 & 40.6\end{array}$ & 7.76 & 1.76 & 0.75 & 0.54 & 11.78 & 11.7 & 11.9 & 0.79 \\
\hline 85 & 20643.4 & $\begin{array}{llll}40 & 20 & 24.2\end{array}$ & 6.93 & 2.01 & 0.68 & 0.58 & 11.24 & 8.7 & 10.2 & 0.67 \\
\hline 86 & $\begin{array}{lll}20 & 19 & 23.7\end{array}$ & $\begin{array}{lll}41 & 44 & 22.0\end{array}$ & 8.63 & 2.23 & 0.67 & 0.61 & 13.18 & 21.0 & 20.9 & 1.15 \\
\hline 87 & $\begin{array}{lll}20 & 19 & 24.4\end{array}$ & $\begin{array}{lll}41 & 43 & 56.3\end{array}$ & 6.57 & 2.04 & 0.60 & 0.60 & 10.93 & 7.5 & 9.6 & 0.41 \\
\hline 88 & $20 \quad 48 \quad 11.2$ & $\begin{array}{lll}36 & 9 & 17.3\end{array}$ & 7.10 & 1.81 & 0.64 & 0.35 & 10.96 & 9.5 & 10.9 & -0.77 \\
\hline 89 & $\begin{array}{lll}20 & 52 & 27.5\end{array}$ & $\begin{array}{lll}36 & 54 & 35.8\end{array}$ & 6.88 & 1.82 & 0.66 & 0.41 & 10.82 & 8.4 & 10.2 & -0.71 \\
\hline 90 & $\begin{array}{lll}21 & 30 & 35.9\end{array}$ & $\begin{array}{lll}44 & 38 & 10.1\end{array}$ & 6.10 & 1.65 & 0.55 & 0.42 & 9.89 & 5.4 & 9.3 & -0.46 \\
\hline 91 & $2141 \quad 11.6$ & 463104.3 & 6.03 & 2.10 & 0.84 & 0.56 & 10.41 & 6.1 & 10.0 & -0.50 \\
\hline 92 & 215209.8 & $\begin{array}{lll}47 & 49 & 13.0\end{array}$ & 6.25 & 1.81 & 0.62 & 0.39 & 10.17 & 6.3 & 10.3 & -0.54 \\
\hline 93 & 215721.6 & $\begin{array}{lll}49 & 53 & 59.1\end{array}$ & 5.50 & 1.75 & 0.55 & 0.44 & 9.41 & 4.3 & 9.2 & -0.29 \\
\hline 94 & $\begin{array}{lll}21 & 58 & 26.3\end{array}$ & $\begin{array}{lll}49 & 38 & 33.7\end{array}$ & 5.11 & 2.03 & 0.68 & 0.46 & 9.32 & 4.0 & 9.0 & -0.29 \\
\hline 95 & 220151.2 & $\begin{array}{lll}50 & 48 & 52.9\end{array}$ & 8.29 & 2.13 & 0.86 & 0.57 & 12.71 & 17.3 & 19.8 & -1.07 \\
\hline 96 & $2203 \quad 44.0$ & $\begin{array}{lll}49 & 46 & 48.9\end{array}$ & 5.28 & 1.88 & 0.66 & 0.43 & 9.31 & 4.1 & 9.1 & -0.33 \\
\hline 97 & 22095.6 & $\begin{array}{llll}50 & 27 & 57.7\end{array}$ & 6.11 & 1.78 & 0.61 & 0.44 & 10.04 & 5.7 & 10.2 & -0.45 \\
\hline 98 & 220930.9 & $\begin{array}{lll}52 & 11 & 30.4\end{array}$ & 5.96 & 1.84 & 0.59 & 0.46 & 9.97 & 5.5 & 10.1 & -0.30 \\
\hline 99 & $22 \quad 1008.7$ & $\begin{array}{lll}51 & 39 & 59.2\end{array}$ & 5.38 & 1.81 & 0.61 & 0.44 & 9.35 & 4.2 & 9.3 & -0.26 \\
\hline 100 & $22 \quad 14 \quad 28.4$ & $\begin{array}{lll}52 & 27 & 41.6\end{array}$ & 5.78 & 1.87 & 0.68 & 0.46 & 9.83 & 5.1 & 9.9 & -0.30 \\
\hline 101 & $22 \quad 18 \quad 54.7$ & $\begin{array}{lll}51 & 39 & 33.2\end{array}$ & 6.17 & 1.70 & 0.62 & 0.29 & 9.87 & 6.0 & 10.5 & -0.46 \\
\hline 102 & $22 \quad 48 \quad 34.6$ & $\begin{array}{lll}54 & 56 & 21.6\end{array}$ & 5.58 & 1.99 & 0.67 & 0.60 & 9.89 & 4.6 & 9.9 & -0.31 \\
\hline 103 & $\begin{array}{lll}23 & 49 & 43.7\end{array}$ & $\begin{array}{lll}57 & 13 & 12.4\end{array}$ & 6.51 & 1.67 & 0.67 & 0.49 & 10.39 & 6.4 & 11.8 & -0.52 \\
\hline
\end{tabular}


Table 4. Radial velocities of C stars.

\begin{tabular}{|c|c|c|c|c|c|c|}
\hline Id & $\ell$ & $\mathrm{b}$ & $V_{\mathrm{h}}$ & err & $V_{\mathrm{LSR}}$ & Notes \\
\hline 1 & 120.8 & -4.8 & -69 & 6 & -70.2 & V382 Cas \\
\hline 2 & 122.3 & -3.2 & -36 & 10 & -37.3 & CGCS 111 \\
\hline 3 & 126.6 & -4.5 & -62 & 5 & -64.3 & V645 Cas \\
\hline 4 & 127.5 & -4.9 & -52 & 2 & -54.5 & \\
\hline 5 & 128.6 & -4.7 & -72 & 8 & -74.7 & \\
\hline 6 & 129.6 & -4.3 & -100 & 2 & -102.9 & \\
\hline 7 & 129.7 & -3.5 & -36 & 10 & -38.8 & V918 Cas \\
\hline 8 & 130.9 & -4.5 & -35 & 10 & -38.1 & CGCS 282 \\
\hline 9 & 131.2 & -4.9 & -82 & 2 & -85.2 & EW Per \\
\hline 10 & 133.1 & -4.5 & -38 & 4 & -41.6 & CN Per \\
\hline 11 & 133.2 & -3.1 & - & - & - & not observed \\
\hline 12 & 135.1 & -4.3 & -64 & 10 & -67.9 & \\
\hline 13 & 136.3 & -4.1 & -35 & 5 & -39.1 & \\
\hline 14 & 133.6 & -3.7 & -25 & 3 & -28.6 & LR Per \\
\hline 15 & 137.1 & -3.8 & -38 & 8 & -42.2 & DY Per \\
\hline 16 & 137.4 & -3.5 & -35 & 4 & -39.2 & CGCS 381 \\
\hline 17 & 160.5 & -4.6 & -38 & 10 & -46.2 & CGCS 702 \\
\hline 18 & 160.8 & -4.6 & -58 & 7 & -66.3 & CGCS 708 \\
\hline 19 & 162.2 & -4.6 & -14 & 4 & -22.5 & CGCS 726 \\
\hline 20 & 161.9 & -3.4 & +51 & 8 & 42.7 & AV Per \\
\hline 21 & 162.1 & -3.3 & -06 & 7 & -14.3 & V395 Per \\
\hline 22 & 171.6 & -3.4 & +49 & 9 & 39.5 & DS Aur \\
\hline 23 & 161.8 & +4.0 & +34 & 12 & 26.7 & CGCS 873 \\
\hline 24 & 176.9 & -3.9 & 0 & 2 & -10.2 & CGCS 916 \\
\hline 25 & 168.9 & +4.0 & -1 & 11 & -9.3 & CGCS 975 \\
\hline 26 & 169.2 & +4.1 & -16 & 9 & -24.3 & CGCS 985 \\
\hline 27 & 190.6 & -4.9 & +45 & 9 & 33.6 & CGCS 1084 \\
\hline 28 & 193.3 & -4.9 & +70 & 11 & 58.5 & \\
\hline 29 & 192.0 & -4.1 & +33 & 12 & 21.6 & \\
\hline 30 & 179.1 & +3.1 & +14 & 2 & 4.5 & CGCS 1107 \\
\hline 31 & 178.3 & +3.9 & -12 & 14 & -21.3 & V508 Aur \\
\hline 32 & 180.1 & +3.8 & +35 & 9 & 25.5 & CGCS 1130 \\
\hline 33 & 181.3 & +3.3 & +33 & 2 & 23.3 & BQ Aur \\
\hline 34 & 181.3 & +3.6 & +40 & 13 & 30.4 & \\
\hline 35 & 197.1 & -4.4 & +59 & 9 & 47.4 & IRAS06032 + 1157 \\
\hline 36 & 199.9 & -4.6 & +51 & 9 & 39.3 & CGCS 6115 \\
\hline 37 & 200.1 & -4.6 & +53 & 13 & 41.3 & CGCS 6117 \\
\hline 38 & 201.0 & -4.1 & +64 & 13 & 52.3 & CGCS 6122 \\
\hline 39 & 202.5 & -4.7 & +43 & 7 & 31.2 & \\
\hline 40 & 202.8 & -4.7 & +36 & 13 & 24.2 & CGCS 1214 \\
\hline 41 & 200.1 & -3.2 & +28 & 9 & 16.4 & V102 \\
\hline 42 & 200.5 & -3.0 & +96 & 3 & 84.4 & CGCS 6129 \\
\hline 43 & 201.9 & -3.4 & - & - & - & not a C star \\
\hline 44 & 203.2 & -3.9 & +34 & 5 & 22.3 & CGCS 1233 \\
\hline 45 & 204.6 & -4.6 & +27 & 9 & 15.2 & CGCS 1236 \\
\hline 46 & 187.2 & +4.8 & +47 & 8 & 37.1 & \\
\hline 47 & 204.2 & -4.1 & +49 & 10 & 37.2 & CGCS 1238 \\
\hline 48 & 203.8 & -3.6 & +66 & 9 & 54.3 & V615 Mon \\
\hline 49 & 204.0 & -3.1 & +71 & 7 & 59.4 & CGCS 6143 \\
\hline 50 & 207.0 & -3.1 & - & - & - & not observed \\
\hline 51 & 196.8 & +3.4 & +38 & 9 & 27.4 & LV Gem \\
\hline 52 & 195.2 & +4.5 & +76 & 11 & 65.6 & CGCS 1311 \\
\hline 53 & 198.3 & +3.6 & - & - & - & not observed \\
\hline 54 & 204.8 & +4.6 & +87 & 6 & 76.3 & CGCS 6186 \\
\hline 55 & 208.1 & +3.0 & +77 & 9 & 66.1 & CGCS 1449 \\
\hline 56 & 206.8 & +3.7 & 101 & 13 & 90.2 & IRAS06508 + 0714 \\
\hline 57 & 204.9 & +4.8 & +83 & 10 & 72.4 & CGCS 1457 \\
\hline 58 & 205.2 & +4.7 & +99 & 11 & 88.3 & IT Mon \\
\hline 59 & 207.9 & +4.1 & - & - & - & not observed \\
\hline 60 & 208.7 & +3.8 & +92 & 11 & 81.2 & CGCS 1484 \\
\hline 61 & 209.4 & +4.4 & - & - & - & not observed \\
\hline 62 & 213.1 & +4.4 & - & - & - & not observed \\
\hline 63 & 215.1 & +4.6 & - & - & - & not observed \\
\hline
\end{tabular}

Table 4. continued.

\begin{tabular}{ccccccl}
\hline \hline Id & $\ell$ & b & $V_{\mathrm{h}}$ & err & $V_{\text {LSR }}$ & Notes \\
\hline 64 & 61.8 & +4.8 & - & - & - & not a C star \\
65 & 62.1 & +4.2 & -63 & 11 & -53.2 & CGCS 6799 \\
66 & 62.4 & +4.3 & -107 & 10 & -97.2 & CGCS 6801 \\
67 & 49.1 & -3.4 & - & - & - & not a C star \\
68 & 63.4 & +4.1 & -145 & 6 & -135.3 & \\
69 & 64.9 & +4.8 & -76 & 6 & -66.4 & CGCS 4366 \\
70 & 65.5 & +4.8 & - & - & - & not a C star \\
71 & 64.0 & +3.7 & - & - & - & not a C star \\
72 & 64.5 & +3.9 & - & - & - & not a C star \\
73 & 52.4 & -3.8 & - & - & - & not a C star \\
74 & 51.2 & -4.6 & - & - & - & not a C star \\
75 & 66.8 & +4.1 & - & - & - & not a C star \\
76 & 66.8 & +4.1 & -58 & 9 & -48.7 & CGCS 4441 \\
77 & 55.1 & -3.6 & - & - & - & not observed \\
78 & 68.5 & +4.5 & -82 & 8 & -72.9 & CGCS 4466 \\
79 & 68.7 & +4.2 & -128 & 10 & -119.0 & CGCS 4481 \\
80 & 56.2 & -3.5 & - & - & - & not a C star \\
81 & 58.4 & -3.7 & - & - & - & not a C star \\
82 & 71.9 & +3.9 & -70 & 11 & -61.4 & \\
83 & 72.8 & +4.0 & - & - & - & not a C star \\
84 & 72.7 & +3.9 & - & - & - & not a C star \\
85 & 76.6 & +4.4 & -76 & 5 & -68.0 & \\
86 & 79.1 & +3.1 & - & - & - & not a C star \\
87 & 79.1 & +3.1 & - & - & - & not a C star \\
88 & 77.9 & -4.7 & -72 & 11 & -65.4 & CGCS 4980 \\
89 & 79.1 & -4.9 & -65 & 17 & -58.6 & V1876 Cyg \\
90 & 89.7 & -.49 & - & - & - & not a C star \\
91 & 92.3 & -4.7 & -93 & 6 & -88.8 & V1732 Cyg \\
92 & 94.6 & -4.9 & -77 & 8 & -73.2 & V1428 Cyg \\
93 & 96.5 & -3.8 & -80 & 6 & -76.4 & V1342 Cyg \\
94 & 96.5 & -4.1 & -66 & 2 & -62.4 & V1410 Cyg \\
95 & 97.7 & -3.5 & - & - & - & not a C star \\
96 & 97.3 & -4.6 & -55 & 5 & -51.7 & \\
97 & 98.4 & -4.5 & -74 & 4 & -70.8 & PU Lac \\
98 & 99.4 & -3.1 & -36 & 6 & -32.8 & CGCS 5592 \\
99 & 99.2 & -3.6 & -82 & 6 & -78.9 & IW Lac \\
100 & 100.2 & -3.3 & - & - & - & PX Lac not a C star \\
101 & 100.3 & -4.4 & - & - & - & LL Lac not a C star \\
102 & 105.8 & -3.8 & -56 & 13 & -54.0 & \\
103 & 114.6 & -4.7 & - & - & - & V1000 Cas not a C star \\
\hline & 7 & & & & & \\
\end{tabular}

